University of Nebraska - Lincoln

DigitalCommons@University of Nebraska - Lincoln

9-1-1985

\title{
A variation-perturbation method for atomic and molecular interactions. II. The interaction potential and van der Waals molecule for $\mathrm{Ne}-\mathrm{HF}$
}

Gordon A. Gallup

University of Nebraska-Lincoln, ggallup1@unl.edu

J. Gerratt

University of Bristol

Follow this and additional works at: https://digitalcommons.unl.edu/physicsgallup

Part of the Physics Commons

Gallup, Gordon A. and Gerratt, J., "A variation-perturbation method for atomic and molecular interactions. II. The interaction potential and van der Waals molecule for Ne-HF" (1985). Gordon Gallup Publications. 10.

https://digitalcommons.unl.edu/physicsgallup/10

This Article is brought to you for free and open access by the Research Papers in Physics and Astronomy at DigitalCommons@University of Nebraska - Lincoln. It has been accepted for inclusion in Gordon Gallup Publications by an authorized administrator of DigitalCommons@University of Nebraska - Lincoln. 


\title{
A variation-perturbation method for atomic and molecular interactions. II. The interaction potential and van der Waals molecule for Ne-HF
}

\author{
G. A. Gallupa) and J. Gerratt \\ Department of Theoretical Chemistry, School of Chemistry, University of Bristol, Bristol BS8 1TS, England
}

(Received 11 February 1985; accepted 26 March 1985)

\begin{abstract}
A recently developed variation-perturbation theory for calculating intermolecular forces has been applied to the $\mathrm{Ne}-\mathrm{HF}$ system for fixed $\mathrm{H}-\mathrm{F}$ distances. The maximum well depth is $0.49 \mathrm{~kJ} /$ $\mathrm{mol}\left(41 \mathrm{~cm}^{-1}\right)$ for a linear configuration with the $\mathrm{H}$ between the $\mathrm{Ne}$ and $\mathrm{F}$ and the $\mathrm{Ne}-\mathrm{H}$ distance approximately $5.5 \mathrm{bohr}$. A secondary minimum of depth $0.24 \mathrm{~kJ} / \mathrm{mol}\left(20 \mathrm{~cm}^{-1}\right)$ was found for the other linear configuration at a Ne-F distance of about $7.0 \mathrm{bohr}$. A saddle point, about $0.20 \mathrm{~kJ} / \mathrm{mol}$ $\left(17 \mathrm{~cm}^{-1}\right)$ deep, for the T-shaped configuration is present also at about $7.0 \mathrm{bohr}$. The potential has been used to calculate some of the bound state internal energies of the Ne-HF van der Waals molecule. The dissociation energy is $15 \mathrm{~cm}^{-1}$ from the lowest state.
\end{abstract}

\section{INTRODUCTION}

In the first article of this series ${ }^{1}$ (hereafter called I) we have developed a general variation-perturbation method for calculating intermolecular forces. In this article we give the results of applying this theory to the calculation of the intermolecular potential between a $\mathrm{Ne}$ atom and a rigid HF molecule. We have also fitted the potential to an analytic expression that can be used to calculate the properties of the van der Waals molecule formed from these two substances.

The van der Waals molecules formed between inert gas atoms and hydrogen halide molecules are an important example of such systems. Ar- $\mathrm{HCl}$ has been the subject of a number of experimental studies ${ }^{2}$ and values for a number of spectroscopic constants have been reported. The He-HF interaction surface has been the subject of a recent theoretical study. ${ }^{3}$ Among these molecules, Ne-HF occupies a unique position, for its properties are such that one expects a stability that will allow it to be studied experimentally without too great a difficulty, while at the same time, with only 20 electrons, it is small enough to expect theory to produce a reasonable accurate surface. We are thus in the position of having a van der Waals molecule that will provide an excellent test of a theory of intermolecular forces.

There have been two other calculations of the $\mathrm{Ne}-\mathrm{HF}$ potential reported in the literature. The calculation of Stone and Hayes ${ }^{4}$ employs a procedure broadly similar to ours in that the total wave function for the system is expanded in a series of antisymmetrized products of wave functions for the individual subsystems. The subsystems are not orthogonalized, and the matrix elements are determined by an expansion in powers of electron interchange. ${ }^{5-9}$ They obtained a potential well of depth $42 \mathrm{~cm}^{-1}$ for $\mathrm{Ne}$ approaching the $\mathrm{H}$ and $5 \mathrm{~cm}^{-1}$ for $\mathrm{Ne}$ approaching the $\mathrm{F}$. They considered collinear geometries only.

Fowler and Buckingham, ${ }^{10}$ using an extremely large basis set, carried out an SCF calculation on the Ne-HF super-

\footnotetext{
a) On leave during the 1983-84 academic year from the University of $\mathrm{Ne}$ braska under the auspices of the S.E.R.C., United Kingdom and the University of Nebraska Research Council. Permanent address: Department of Chemistry, University of Nebraska, Lincoln, Nebraska 68588.
}

molecule for collinear geometries. The Ne-HF well depth is $11 \mathrm{~cm}^{-1}$ and that for Ne-FH is less than $1 \mathrm{~cm}^{-1}$. Such a calculation includes induction and charge transfer effects but no dispersion. However, its purpose was to examine the nature of the basis set superposition error and in particular, to show that the usual correction by means of the Boys counterpoise methods serves only to introduce further unphysical effects.

We have used our potential in a preliminary determination of the bound states of $\mathrm{Ne}^{22}-\mathrm{HF}$. This isotopic species does not have a mass coincidence that complicates experiments. However, most of the internal states of Ne-HF are quasi-bound, and a detailed study of these will be reported in a subsequent publication.

\section{DETAILS OF CALCULATION}

\section{A. The $A O$ basis}

Table I gives the Slater basis used for each atom. It will be seen to be between double- $\zeta$ and triple- $\zeta$-plus-polarization functions in quality. The original double- $\zeta$ set is taken from Clementi and Roetti. ${ }^{11}$ The third $2 s$ and $2 p$ and the $3 d$ orbitals on $\mathrm{Ne}$ have their scale factors adjusted to optimize

TABLE I. Slater orbital basis sets for $\mathrm{Ne}, \mathrm{H}$, and $\mathrm{F}$.

\begin{tabular}{|c|c|c|c|c|c|c|c|c|}
\hline \multicolumn{3}{|c|}{$\mathrm{Ne}^{\mathrm{a}}$} & \multicolumn{3}{|c|}{ H } & \multicolumn{3}{|c|}{$F^{a}$} \\
\hline$n$ & $l$ & & $n$ & $l$ & & $n$ & $l$ & \\
\hline 1 & 0 & 11.4216 & 1 & 0 & 1.0 & 1 & 0 & 10.4245 \\
\hline 1 & 0 & 8.50182 & 1 & 0 & 1.3 & 1 & 0 & 7.66585 \\
\hline 2 & 0 & 3.56883 & 2 & 1 & 1.0 & 2 & 0 & 3.13578 \\
\hline 2 & 0 & 2.19285 & & & & 2 & 0 & 1.44456 \\
\hline 2 & 0 & $1.256^{\mathrm{b}}$ & & & & 2 & 1 & 4.18389 \\
\hline 2 & 1 & 4.68773 & & & & 2 & 1 & 1.85062 \\
\hline 2 & 1 & 2.05684 & & & & 3 & 2 & $2.50^{\mathrm{c}}$ \\
\hline 2 & 1 & $0.759^{\mathrm{b}}$ & & & & & & \\
\hline 3 & 2 & $1.82072^{\mathrm{b}}$ & & & & & & \\
\hline
\end{tabular}

a The orbitals unmarked with superscripts are the double- $\zeta$ basis sets from Ref. 11.

${ }^{b}$ These values were determined to maximize the calculated polarizability of Ne.

${ }^{\mathrm{C}}$ This scale factor gives the correct dipole moment to the SCF function for HF. 
the Ne polarizability determined using the functional of I [Eq. (8)].

The $3 d$ orbital set on $\mathrm{F}$ has its scale factor adjusted so that the SCF function for HF yields the correct experimental value for the electric dipole moment. This produces an increase of only 0.0002 a.u. in the energy above the optimum SCF energy possible with this basis. This discrepancy is not expected to influence the calculated potential because of the systematic cancellation of errors of the type discussed in $I$.

The orbital scale factors of the $\mathrm{H}$ atom conform to the commonly used values for $\mathrm{H}$ attached to $\mathrm{F}$.

\section{B. Pseudo-state orbitals and level shifts}

The experimental value for the polarizability of $\mathrm{Ne}$ is $2.67 \mathrm{bohr}^{3} \cdot{ }^{12}$ Without a level shift, the basis of Table I gives a polarizability of $2.37 \mathrm{bohr}^{3}$, which is about $11 \%$ low. The level shift required to make the calculation match experiment is -0.150 a.u.

In our calculations the Ne $1 s$ orbital was kept doubly occupied in all configurations. This leads to a set of pseudostate orbitals for Ne that we denote as $3 s, 3 p$, and $3 d$. With the high symmetry of the $\mathrm{Ne}$ atom only one orbital set of each angular momentum is required.

The spherically averaged polarizability of HF has apparently not been measured, although the anisotropy $\alpha_{\|}-\alpha_{1}$ has been given as 1.620 bohr $^{3} .{ }^{13}$ Lipscomb $^{14}$ has given theoretical values of 5.803 and 4.183 bohr $^{3}$ for $\alpha_{\|}$and $\alpha_{1}$, respectively, and these values accurately reproduce the difference. With the basis of Table $I$, and no shift correction, we calculate $\alpha_{\|}$and $\alpha_{1}$ values of 4.197 and 3.116 bohr $^{3}$, respectively. The required level shift is the same for both tensor components and is -0.123 a.u. The level shift increased the calculated polarizability by $25 \%$ (see Ref. 15 ).

In our calculations the HF $1 \sigma$ orbital was kept doubly occupied in all configurations. This gives rise to a pseudostate orbital set on HF denoted by $4 \sigma, 5 \sigma, 6 \sigma, 2 \pi, 3 \pi$, and $1 \delta$. It is seen that both $\mathrm{Ne}$ and $\mathrm{HF}$ require nine pseudo-state orbitals, but the higher symmetry of Ne produces a set with less duplication of symmetry species.

\section{Classification and calculation of integrals}

In I we categorized the two electron integrals as "onboard" and "intersystem" types. It is useful to give a somewhat finer classification with general formulas for the numbers of each type. If we have a van der Waals system with two subsystems, $a$ and $b$, having $n_{a}$ and $n_{b}$ orbitals in their descriptions, there are

$$
p_{a}\left(p_{a}+1\right) / 2+p_{b}\left(p_{b}+1\right) / 2
$$

on-board integrals, where $p_{i}=n_{i}\left(n_{i}+1\right) / 2$, and $i=a, b$.

The intersystem types can be classified as generalized Coulomb, generalized hybrid, and generalized exchange. These terms are related to the names traditionally used for diatomic molecules. The generalized Coulomb integrals fall off asymptotically as some inverse power of $R$. There are, in general, $p_{a} p_{b}$ of these.

The generalized hybrid types involve a pair of orbitals on one subsystem interacting with the product of two orbitals, one from each subsystem. There are $\left(p_{a}+p_{b}\right) n_{a} n_{b}$ of this type.
The last type consist of the generalized exchange integrals. There are $n_{a} n_{b}\left(n_{a} n_{b}+1\right) / 2$ of these.

For the calculation described here, with 19 orbitals on Ne and 20 orbitals on HF, these formulas give
40300
on-board,
39900 generalized Coulomb,
152000 generalized hybrid,
72390 generalized exchange

types. This is a total of 304590 possible two electron integrals, of which a relatively small number are normally zero.

The on-board integrals were calculated with the Gaussian transform method using the Stevens integral package, POLYCAL. ${ }^{16}$ As was emphasized in I, this was done only once and took only a few minutes on an IBM 3081 computer.

Referring again to I, we used the multipole expansion method for the generalized Coulomb intergrals. For these calculations the expansions were taken as far as quadrupole terms. This is sufficient to give values of the integrals typically within $0.01 \%$ of those from the Stevens program. On the IBM 3081 machine the 39900 integrals of this type could be calculated in 4-6s.

The generalized hybrid and exchange integrals constitute $74 \%$ of the total in our system, and it is clearly important to calculate them efficiently. The Fourier transform approximation scheme of $I$ was used on both these types of integrals. The numerical quadrature formulas involved were implemented with four quadrature points in the $k$ and the $\theta_{k}$ directions and eight in the $\phi_{k}$ direction. These integrals are typically accurate within $1 \%$ of the value given by the Stevens program and required $3-4 \mathrm{~min}$ on the IBM $3081 \mathrm{com}$ puter.

\section{Cl calculation}

The orbital basis set used in the calculations was as follows:

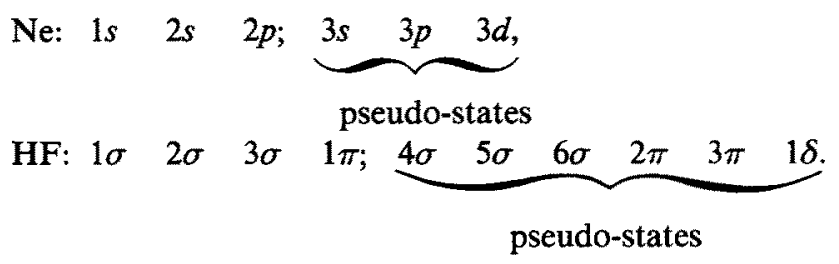

It should be recalled that the $\mathrm{Ne}$ and HF sets are not orthogonal to one another, and are used directly in the VB calculation.

The $1 s$ and $1 \sigma$ orbitals were kept doubly occupied throughout. Inclusion of all single and double excitations of the types discussed below from the 14 remaining orbitals gives rise to 720 configurations of $A^{\prime}$ symmetry (even with respect to a reflection in the plane of the system). These configurations may be grouped into three classes as described in I and give rise to the following contributions to the intermolecular potential:

(ia) Single excitations; occupied pseudo-state orbital on $\mathrm{Ne}$ atom: Polarization of Ne by HF.

(ib) Single excitations; occupied pseudo-state orbital on HF molecule: Polarization of HF by Ne.

(ii) Double excitations; occupied $\mathrm{Ne}$ pseudo-state $\mathrm{Ne}$; occupied HF pseudo-state HF: dispersion interaction. 
TABLE II. The intermolecular potential of Ne-HF at 50 points. (Energies in $\mathrm{m}$ hartrees). The asymptotic energy is - 228.59062154 a.u.

\begin{tabular}{cccccc}
\hline \hline & \multicolumn{4}{c}{$\theta$ (deg) } \\
\cline { 2 - 6 } & 0.0 & 45.0 & 90.0 & 135.0 & 180.0 \\
\hline 20.0 & -0.0017089 & -0.0010780 & -0.0001334 & -0.0001823 & -0.0001943 \\
15.0 & -0.0164098 & -0.0190840 & -0.0010068 & -0.0090210 & -0.0089687 \\
12.0 & -0.0281769 & -0.0314167 & -0.0216049 & -0.0221777 & -0.0186637 \\
10.0 & -0.0503485 & -0.0533434 & -0.0343379 & -0.0407525 & -0.0343502 \\
9.0 & -0.0779462 & -0.0683273 & -0.0474400 & -0.0482968 & -0.0476991 \\
8.0 & -0.1329275 & -0.0972377 & -0.0576612 & -0.0613196 & -0.0617196 \\
7.5 & -0.1767283 & -0.1064412 & -0.0574052 & -0.0640349 & -0.0742065 \\
7.0 & -0.1896082 & -0.1065558 & -0.0486890 & -0.0490468 & -0.0743224 \\
6.5 & -0.1121432 & -0.0732230 & -0.0129399 & 0.0217504 & 0.0455650 \\
6.0 & 0.05605102 & -0.0027279 & 0.0551343 & 0.2683930 & 0.2601761 \\
\hline
\end{tabular}

The accuracy of the quadrature formulas used in obtaining the generalized hybrid and exchange integrals was insufficient for this point and the Stevens program was used. This point fits smoothly into the others with no difficulty, however.

(iiia) Single excitation; occupied Ne pseudo-state HF; $\mathrm{Ne}^{+} \mathrm{HF}^{-}$charge transfer.

(iiib) Single excitation; occupied HF pseudo-state Ne: $\mathrm{Ne}^{-} \mathrm{HF}^{+}$charge transfer. There are further double excita-
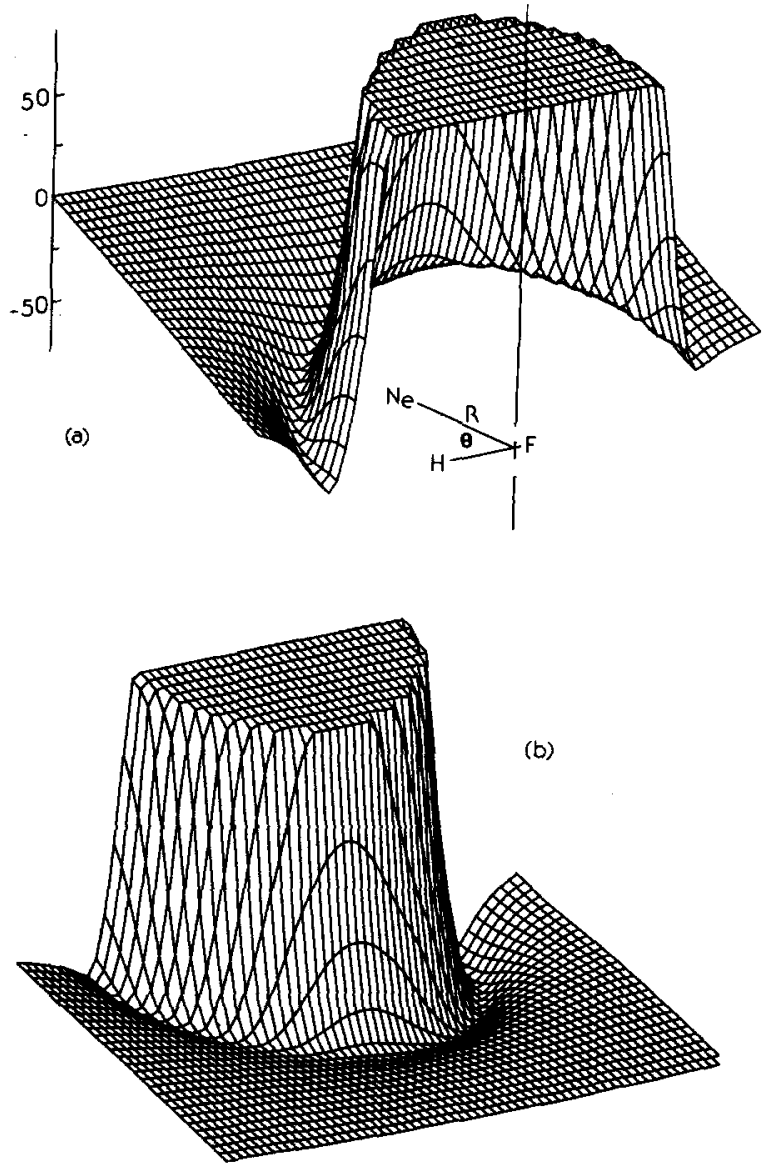

FIG. 1. Two different views of a pseudo-perspective three-dimensional representation of the intermolecular potential between $\mathrm{Ne}$ and HF. In each case half of the surface is shown. (a) is a section through the HF molecule with a stick drawing showing the relation of the coordinates of the atom positions to the surface. The "front edge" of the surface shows the potential along the two possible linear configurations. The vertical scale is in $\mathrm{cm}^{-1}$. (b) is the same surface seen from the "backside." In both cases the portion of the surface where the two molecules are approaching closely to one another is truncated at $100 \mathrm{~cm}^{-1}$. tions within $\mathrm{Ne}$ along and within $\mathrm{HF}$ alone that provide correlation effects within the isolated subsystems. Such configurations were not included in the present calculations. The use of the level shift technique compensates-at least partial$1 y$-for their absence as far as the intermolecular interaction is concerned. No charge transfer excitations [(iiia), (iiib)] were included either. The omission of these configurations is discussed below.

The double excitations (ii), which describe the dispersion interactions, are all of the form:

$$
\left\{1 \sigma^{2} 2 \sigma^{2} 3 \sigma^{2} 1 \pi^{3} 4 \sigma\left({ }^{1} \Pi ;{ }^{3} \Pi\right) ; 1 s^{2} 2 s^{2} 2 p^{5} 3 s\left({ }^{1} P ;{ }^{3} P\right)\right\},
$$

i.e., both singlet-singlet and triplet-triplet couplings between $\mathrm{HF}$ and $\mathrm{Ne}$ are included for each case.

The Hamiltonian and overlap matrices were evaluated for the functions, and the potential of the interaction was obtained directly according to the procedure described in $\mathbf{I}$.

The potential is expressed in terms of the distance between the two subsystems $R$ measured from the center of mass of normal isotope HF to the Ne nucleus and the angle $\theta$ measured from the $\mathrm{H}$ end of the molecule. We have calculated energies for 50 different geometries: $R=6.0,6.5,7.0,7.5$, $8.0,9.0,10.0,12.0,15.0$, and $20.0 \mathrm{bohr}$ for each of the angles, $\theta=0.0,45.0,90.0,135.0$, and $180.0 \mathrm{deg}$. The specific values are given in Table II.

\section{INTERMOLECULAR POTENTIAL BETWEEN Ne AND HF}

The energies of Table II have been fitted to an expression of the form

$$
V(R, \theta)=\sum_{l}^{\prime} V_{l}(R) P_{l}(\cos \theta)
$$

for values of $l$ up to 4 . The $V_{l}(R)$ have been represented by exponential functions, splines, and inverse powers of $R$ depending upon the range. A general purpose FORTRAN function subprogram that gives this potential has been written and is available from the authors.

The depth of the potential in the $\theta=0$ direction is 41.6 $\mathrm{cm}^{-1}$, in the $\theta=90$ direction it is $17.0 \mathrm{~cm}^{-1}$, and in the $\theta=180$ direction it is $20.4 \mathrm{~cm}^{-1}$. A simulated three-dimensional altitude drawing of the fitted energies is shown in Fig. 1. 


\section{BOUND STATES OF THE NE-HF VAN DER WAALS MOLECULE}

We have used the potential $V(R, \theta)$ of Eq. (5) to carry out a preliminary calculation of the bound states of the $\mathrm{Ne}^{22}-\mathrm{HF}$ system. The wave functions are expressed as

$$
\psi_{n}^{J M}=\sum_{j l} f_{j l n}^{J}(R) \phi_{j l}^{J M}(\omega, \Omega) .
$$

In this equation $\phi_{j l}^{J M}(\omega, \Omega)$ is an angular function in which the orbital angular momentum of Ne about $\mathrm{HF}, l$, is coupled with the rotational angular momentum of $\mathrm{HF}, j$, to give the resultant $J$ :

$$
\phi_{j l}^{J M}=\sum_{m_{j}, m_{l}}\left\langle j l m_{j} m_{l} \mid J M\right\rangle Y_{j m_{j}}(\omega) Y_{l m_{l}}(\Omega) .
$$

The angular coordinates $\omega$ and $\Omega$ refer to space fixed axes.

The radial functions $f_{j i n}^{J}(R)$ are determined by an expansion in a basis set of harmonic oscillator functions, according to the procedure of Bratoz and Martin. ${ }^{17}$

These calculations predict two bound states in the well. The energies are shown in Table III and Fig. 2 for $J=0,1$, and 2. The value of $D_{0}$ is $14.7 \mathrm{~cm}^{-1}$, and the zero point energy is $26.3 \mathrm{~cm}^{-1}$.

The value of $B_{e}$ for $\mathrm{HF}$ is $20.939 \mathrm{~cm}^{-1,18}$ and consequently all states of Ne-HF where HF is executing internal rotations with respect to the Ne-HF framework will be quasi-bound. A more detailed study of the energies and the lifetimes of these states will be reported in a subsequent publication. ${ }^{19}$

The pattern of the bound state energy levels is shown in Fig. 2. These are what one would expect for a linear molecule and are easily fitted to the formula

$$
E_{n J} / h c=B_{n} J(J+1)-D_{n}[J(J+1)]^{2}
$$

with

$$
\begin{aligned}
& B_{0}=0.106 \mathrm{~cm}^{-1}, D_{0} \leqslant 5 \times 10^{-4} \mathrm{~cm}^{-1}, \\
& B_{1}=0.08 \mathrm{~cm}^{-1}, D_{1}=0 .
\end{aligned}
$$

A sizable effect of an excitation of the Ne-HF stretching motion is evident.

\section{E. Discussion}

The present calculations show that the Ne-HF potential has a well-depth of $41.6 \mathrm{~cm}^{-1}$ in the collinear Ne-H-F configuration and a secondary well $20.4 \mathrm{~cm}^{-1}$ deep in the $\mathrm{Ne}-\mathrm{F}-\mathrm{H}$ configuration. The pattern of the energies of the bound levels indicates that the motion of $\mathrm{Ne}-\mathrm{HF}$ is almost completely linear.

As explained in I, the purpose of the level shift correlation is to yield the correct dipole polarizabilities for both $\mathrm{Ne}$ and HF. A small adjustment of the exponent of the $3 d$ basis function of $\mathrm{F}$ also provides the correct dipole moment of $\mathrm{HF}$.

TABLE III. Energies of Ne-HF van der Waals molecule (in $\mathrm{cm}^{-1}$ ).

\begin{tabular}{ccc}
\hline \hline$L$ & $k=0$ & $k=1$ \\
\hline 0 & -14.72 & -5.47 \\
1 & -14.52 & -5.31 \\
2 & -14.14 & -4.99 \\
\hline
\end{tabular}

\section{SIX ENERGY STATES OF Ne-HF}
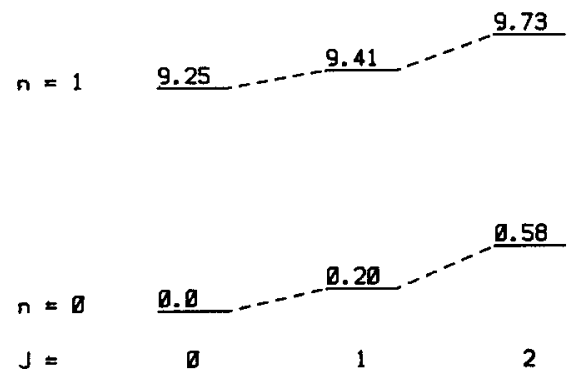

FIG. 2. Pattern of energy levels of the bound states of Ne-HF. The energies are given in $\mathrm{cm}^{-1}$.

Consequently we are reasonably confident that the $R^{-6}$ part of the potential, which arises from induction and dispersion effects, is accurate.

The succeeding terms in $R^{-7}$ and $R^{-8}$, which respectively arise from dipole-quadrupole induction and quadrupole-quadrupole induction + quadrupole dispersion interactions, although present in the calculations [as excitations (ia) and (ii)], have not been optimized in the same way. We regard this as the most significant shortcoming of our calculation.

Our calculations do not include any correlation effects on the isolated Ne and HF subsystems (double excitations for each subsystem, giving rise to double + quadrupole excitations in the interacting system). As argued above the level shifts compensate at least partially for this absence as far as intermolecular interactions are concerned. In several previous studies the $\mathrm{MO}-\mathrm{CI}$ approach have focused on the interaction between intramolecular (subsystem) correlation and intermolecule (dispersion) correlation, ${ }^{20}$ and conclude that this is significant. A careful study of $\mathrm{He}-\mathrm{He}$ using VB theory ${ }^{21}$ found on the contrary that there is no significant interaction between these two types of correlation. It would appear that, at least for $\mathrm{He}-\mathrm{He}$, the manifestation of an interaction between the two types of correlation is an artifact of a particular model. If this turns out to be generally true, it would represent a useful additional feature of the VB approach.

The absence of charge transfer (CT) configurations in our calculation ensures that there can be no basis set superposition error. The straightforward inclusion of CT in VB calculations is known to produce this error, ${ }^{21,22}$ and can result in potential wells that are far too deep. The essential cause of this [for excitations of type (iiia) and (iiib)] is that CT configurations provide spurious improvements in the descriptions of the subsystems as they approach one another. With well optimized subsystem orbitals this type of error is completely missing, and we are confident of having approached this level of accuracy.

In the present case if one considers genuine single excitation CT affects, this would mean the inclusion of the configurations pertaining to the structures $\mathrm{Ne}^{+}, \mathrm{HF}^{-}$and $\mathrm{Ne}^{-}$, 
$\mathrm{HF}^{+}$. Simply to state these is to emphasize how negligible such contributions are likely to be. Although $\mathbf{H F}^{-}$is stable, the disparity between its electron affinity and the ionization potential of $\mathrm{Ne}$ indicates that $\left\{\mathrm{Ne}^{+} \mathrm{HF}^{-}\right\}$configurations may be safely neglected. When we consider the other direction of CT we see that all states of $\mathrm{Ne}^{-}\left(2 p^{6},{ }^{1} S ; n l ;{ }^{2} l\right)$ are metastable and strongly coupled to the continuum. This and the ionization potential of HF indicate that $\mathrm{Ne}$ has no particular interest in another electron from HF and these configurations can also be safely neglected.

More complicated CT configurations (double and higher excitations with charge transfer) produce a basis set superposition error that results from a spurious increase in the intramolecular correlation as the subsystems approach one another. This error could be effectively eliminated if the subsystems were thoroughly correlated by other configurations. However, this is impractical in a system of the size we are discussing here, and we have not included any CT configurations of the type that cause this difficulty.

Our present results for the $\mathrm{Ne}-\mathrm{H}-\mathrm{F}$ well depth $(41$ $\mathrm{cm}^{-1}$ ) are in almost perfect agreement with the results of Stone and Hayes. ${ }^{4}$ There is, however, a considerable discrepancy for the Ne-F-H well, which we find to be $\sim 4$ times deeper $\left(20 \mathrm{~cm}^{-1}\right.$ compared to $\left.5 \mathrm{~cm}^{-1}\right)$. The Stone and Hayes calculations made use of considerably smaller basis sets than the present work, a fact that almost certainly accounts for this disagreement.

It is perhaps worthwhile pointing out the extreme long range of the $\mathrm{Ne}-\mathrm{HF}$ interaction. For $R(\mathrm{Ne}-\mathrm{HF})=20 a_{0}$, the potential is still $\sim-0.4 \mathrm{~cm}^{-1}$. This conforms to features of potentials calculated with semiempirical HF (HartreeFock) + damped dispersion procedure, but is not usually found in $a b$ initio calculations. While the $a b$ initio work does indeed possess the correct long range $R^{-n}$ character, the potentials generally fall off too rapidly. This shortcoming, it seems to us, can be attributed to the use of too few long range Gaussian basis functions, so that the tails of these wave functions fall off too rapidly.
Our potential indicates that the H end of HF is "softer" to Ne approach than the F end. We attribute the larger depth of the well there to a lower electron density and a lower repulsion that allows the $\mathrm{Ne}$ to approach the $\mathrm{H}$ end more closely so that the attractive part of the potential can become larger before being cut off by the repulsive part. One result of this is that the geometric surface in space defined by $(\partial \mathrm{V} /$ $\partial r)=0$ is surprisingly close to spherical with the center at the normal isotopic HF center of mass. Because this center of mass is very close to the $F$ nucleus, Ne can approach the $\mathbf{H}$ rather more closely than it does the $\mathrm{F}$.

${ }^{1}$ G. A. Gallup and J. Gerratt, J. Chem. Phys. 83, 2316 (1985).

${ }^{2}$ S. E. Novak, S. J. Harris, K. C. Janda, and W. Klemperer, Can. J. Phys. 53, 2007 (1975).

${ }^{3}$ M. Raimondi, Mol. Phys. 53, 161 (1984).

${ }^{4}$ A. J. Stone and I. C. Hayes, Faraday Discuss. Chem. Soc. 73, 19 (1984).

5J. N. Murrell, M. Randic, and D. R. Williams, Proc. Soc. London Ser. A 284, 566 (1965).

${ }^{6}$ P. Dacre and R. McWeeny, Proc. Soc. London Ser. A 317, 435 (1970).

7J. Gerratt, Proc. R. Soc. London Ser. A 350, 363 (1976).

${ }^{8}$ J. Gerratt and M. Papadopolous, Mol. Phys. 41, 1071 (1980).

${ }^{9}$ I. C. Hayes and A. J. Stone, Mol. Phys. 53, 69, 89 (1984).

${ }^{10}$ P. W. Fowler and A. D. Buckingham, Mol. Phys. 50, 1349 (1983).

${ }^{11}$ E. Clementi and R. S. Roetti, At. Data Nucl. Data Tables 14, 428 (1974).

${ }^{12}$ R. R. Teachout and R. R. Pack, At. Data 3, 195 (1971).

${ }^{13}$ J. S. Muenter, J. Chem. Phys. 56, 5409 (1972).

${ }^{14}$ W. N. Lipscomb, Adv. Magn. Reson. 2, 137 (1966), Table V.

${ }^{15}$ G. A. Gallup and J. Gerratt, Chem. Phys. Lett. 112, 228 (1984).

${ }^{16}$ R. M. Stevens, POLYCAL, multicenter integrals over Slater orbitals by the Gaussian transform method, Harvard University (1979).

${ }^{17}$ S. Bratoz and M. L. Martin, J. Chem. Phys. 42, 1051 (1965).

${ }^{18}$ K. P. Huber and G. Herzberg, Molecular Spectra and Molecular Structure. IV. Constants of Diatomic Molecules (Van Nostrand Reinhold, New York, 1979).

${ }^{19} \mathrm{~J}$. Gerratt and G. A. Gallup (to be submitted).

${ }^{20}$ B. Liu and A. D. MacLean, J. Chem. Phys. 59, 4557 (1973); J. Schaefer and W. Meyer, ibid. 70, 344 (1979).

${ }^{21}$ J. R. Collins and G. A. Gallup, Mol. Phys. 49, 871 (1983).

${ }^{22}$ D. Chen, J. R. Collins, and G. A. Gallup, J. Chem. Phys. (submitted for publication). 